\title{
Identification of the External Branch of the Superior Laryngeal Nerve: An Additional Argument for Neuromonitoring?
}

\author{
Peter Angelos, MD, PhD, FACS \\ The University of Chicago Medicine, Chicago, IL
}

In contrast to the less well-known external branch of the superior laryngeal nerve (EBSLN), the recurrent laryngeal nerve (RLN) has been well-known to anatomists and surgeons for centuries. Although Leonardo da Vinci may have been the first to demonstrate the RLN in his drawings from 1503 , Galen was apparently the first to show that dividing the RLN in a pig resulted in the pig's squealing to cease. ${ }^{1}$ Despite knowledge of the anatomy and function of the RLN that surgeons had for centuries, patients continued to experience the morbidity of RLN injuries after thyroidectomy. In Billroth's series of thyroidectomies between 1877 and $1881,25 \%$ of patients had unilateral RLN injuries and $4.5 \%$ suffered bilateral injuries. ${ }^{1}$

The problem of RLN injuries after thyroidectomy led to many approaches to solve the problem. Among the most influential was the strategy recommended by George Crile in his influential 1932 textbook entitled Diagnosis and Treatment of Diseases of the Thyroid Gland. Crile advocated avoiding injury to the nerve by avoiding exposure of it. He specifically argued for leaving the posterior capsule of the thyroid gland so that the RLN was never exposed during the course of a thyroidectomy. ${ }^{2}$ This approach to the technique of thyroidectomy was commonly used throughout much of the early 20th century. Despite the influence of this approach of avoidance of the nerve, Lahey in $1938^{3}$ and subsequently Riddell in $1956^{4}$ showed excellent results by attempting to identify

(C) Society of Surgical Oncology 2015

First Received: 9 November 2014; Published Online: 7 February 2015

P. Angelos, MD, PhD, FACS

e-mail: pangelos@surgery.bsd.uchicago.edu and then protect the RLN in every case. In recent decades, this approach of identification in order to protect the RLN has become widely used and has been shown to result in lower rates of RLN injury. ${ }^{5}$

Why, one might ask, is the history of the RLN relevant in commenting on the study by Glover and colleagues on the use of neuromonitoring to aid in identification of the EBSLN? ${ }^{6}$ I would argue that today the EBSLN is treated in much the same way that surgeons used to routinely treat the RLN—namely, "don't worry about finding it as long as you avoid injuring it". Nevertheless, many authors have shown that with a concerted effort to identify the EBSLN, they can be found in a majority of cases. However, the accuracy of the identification has often been suspect since other structures can look like the nerve (muscle fibers, connective tissue, blood vessels), and postoperative assessment of EBSLN is more complicated than simply seeing the vocal cords move during laryngoscopy.

Glover and colleagues have shown that the use of neuromonitoring techniques can improve the already high rate of EBSLN identification by a group of very experienced and high-volume endocrine surgeons. These authors found that by utilizing initial dissection and visualization techniques, $85.7 \%$ of 357 nerves at risk were correctly identified. However, that rate went up to $97.2 \%$ of nerves at risk when visualization was confirmed with neuromonitoring and confirmation of a cricothyroid twitch. If neuromonitoring can help this group of surgeons improve their rates of successful EBSLN identification, consider how valuable the technique may be for the low-volume or inexperienced thyroid surgeon. Although only time will tell, could we be on the verge of realizing that routine identification of the EBSLN is a safer strategy than simply avoidance? If the history of the RLN is in any way applicable, finding and protecting a nerve may be a better way to ensure its function rather than simply trying to avoid it. 


\section{REFERENCES}

1. Kaplan EL, Salti GI, Roncella M, et al. History of the recurrent laryngeal nerve: from Galen to Lahey. World J Surg. 2008;33: 386-93.

2. Crile $\mathrm{G}$ et al. Diagnosis and treatment of diseases of the thyroid gland. Philadelphia: W.B. Saunders; 1932. p. 401-09.

3. Lahey F. Routine dissection and demonstration of the recurrent laryngeal nerve in subtotal thyroidectomy. Surg Gynecol Obstet. 1938;66:775-77.

4. Riddell V. Injury to recurrent laryngeal nerves during thyroidectomy: a comparison between the results of identification and non- identification in 1022 nerves exposed to risk. Lancet. 1956;29: 638-41.

5. Hermann M, Alk G, Roka R, et al. Laryngeal recurrent nerve injury in surgery for benign thyroid diseases: effect of nerve dissection and impact of individual surgeon in more than 27,000 nerves at risk. Ann Surg. 2002;235:261-68.

6. Glover AR, Norlen O, Gundara JS, et al. Use of the nerve integrity monitor during thyroid surgery aids identification of the external branch of the superior laryngeal nerve. Ann Surg Oncol. doi:10. 1245/s10434-014-4142-3. 